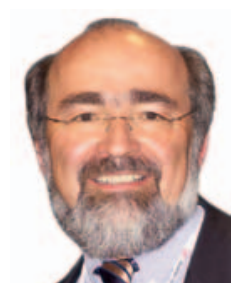

Prof. Dr. Helmut Schöfer

schoefer@em.uni-frankfurt.de

Die Angst vor AIDS mit seinen schweren Begleiterkrankungen und dem (unbehandelt) letalen Ausgang hatte erhebliche Auswirkungen auf die Epidemiologie anderer sexuell übertragbarer Infektionen (STI). Syphilis, Gonorrhö und vermutlich auch alle anderen STI waren durch Vorsicht bei der Partnerwahl und Zunahme des Kondomgebrauchs (Safer Sex) in den 1980er und 1990er Jahren stark zurückgegangen. In Deutschland wurde die Syphilis über ein Jahrzehnt mit einer «historisch» niedrigen Inzidenz von jährlich 2 Fällen/100 000 Einwohner gemeldet. Etwa seit dem Jahr 2000 sehen wir jedoch eine rasante Wiederkehr dieser Erkrankung (Inzidenz 2012: 5,4 Fälle/ 100000 Einwohner; zu >80\% waren Männer betroffen, die Sex mit Männern hatten). Ebenso ist seither eine Zunahme der HIVNeuinfektionen und - sozusagen huckepack - anderer STI zu verzeichnen.

Daher wird es für uns Dermatologen wieder wichtiger, uns daran zu erinnern, dass wir die Berufsbezeichnung «Dermatovenerologe» erworben haben und vor allem unser Berufsstand für die klinische Diagnostik und Therapie der vielgestaltigen STI verantwortlich ist. Ein Blick in die Lehrbücher in Hinblick auf - mangels Bedarf im klinischen Alltag - in Vergessenheit geratenes venerologisches Fachwissen und in die aktuellen Leitlinien (bei AWMF online oder auf der Homepage der DSTIG) zur Aktualisierung der Therapiekonzepte ist zu empfehlen. Auch in der «braven» Landgemeinde kön-

\title{
Sexuell übertragbare Infektionen - Neues zu fast vergessenen Erkrankungen
}

nen Ihnen heute wieder Syphilis \& Co. begegnen. Die beiden Übersichtsarbeiten dieses Hefts beschäftigen sich daher mit zwei klinisch relevanten STI-Themen: Genitale Chlamydien-Infektionen verlaufen häufig stumm und werden leicht übertragen. Auch bei unbemerkter Infektion besteht die Gefahr, dass Infertilität oder schwere Komplikationen auftreten. Hinzu kommt eine ganz überwiegend bei homosexuellen Männern auftretende Infektion mit den Chlamydien-Serovaren L1-3, das Lymphogranuloma venereum. Die differenzierte Therapie der Chlamydien-Infektionen wird im ersten der beiden Übersichtsartikel besprochen. Im zweiten Übersichtsreferat geht es um die Krebsvorsorgeuntersuchung für das Zervixkarzinom. Mehrere Studien haben gezeigt, dass das genitale Primär-Screening auf onkogene humane Papillomviren (HPV) mittels der Polymerase-Kettenreaktion der bisherigen Standardmethode (Zytologie nach Papanicolaou) ebenbürtig bis überlegen ist. Vor allem bei HPV-positiven Frauen über 30 Jahre kann die HPV-Diagnostik, im positiven Fall ergänzt durch eine p16-Ki67-Diagnostik, den bisherigen Standard ersetzen.

Die Rubrik Wissenstransfer beinhaltet Übersetzungen von insgesamt 10 Abstracts wichtiger wissenschaftlicher Arbeiten nebst Kommentaren zur praktischen Relevanz dieser Artikel durch unsere Experten. Diagnostisch relevante Themen wie die konfokale Reflexionsmikroskopie am Basal- zellkarzinom, aber auch aktuelle Untersuchungen zur Psoriasis-Therapie, neue therapeutische Verfahren zur Wundheilung und vieles mehr werden diskutiert. So werden auch in diesem Heft viele Facetten des breit angelegten Fachgebiets Dermatologie berücksichtigt.

In der Rubrik Erfahrungen aus der Praxis finden Sie in diesem Heft zwei Kasuistiken. Nach der Behandlung einer frontalen fibrosierenden Alopezie mit dem 5a-Reduktasehemmer Dutasterid und Pimecrolimus traten diffuse Hyperpigmentierungen auf. In dem anderen Fallbeispiel zeigte eine autosomal-rezessive Hypotrichose mit Kräuselhaar bei einem Kind einen wechselhaften Verlauf über die ersten 3 Lebensjahre. Die genetische Störung konnte als homozygote Mutation auf Exon 6 des Lipase-H-Gens identifiziert werden. Beide Eltern waren heterozygote Träger der gleichen Mutation.

Soviel als «Appetithäppchen» zu dieser spannenden Ausgabe des Karger Kompass Dermatologie. Ihnen, lieber Leser, viel Spaß bei der Lektüre!

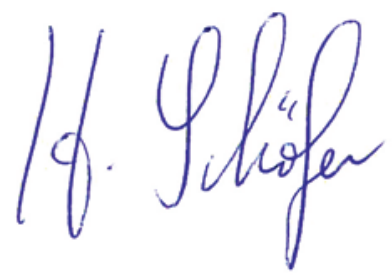

\section{KARGER}

Fax +4976145207 14

information@karger.com www.karger.com (c) 2014 S. Karger GmbH, Freiburg

2296-5424/14/0022-0061\$39.50/0

Accessible online at:

www.karger.com/kk
Prof. Dr. Helmut Schöfer

Klinik für Dermatologie, Venerologie und Allergologie

Klinikum der Johann Wolfgang Goethe-Universität

Theodor-Stern-Kai 7, 60590 Frankfurt, Deutschland 